\title{
The Usefulness of Magnetic Resonance Imaging in the Preoperative Study and Postoperative Control in the Laparoscopic Treatment of the Incisional Hernia
}

\author{
J. C. Martín del Olmo ${ }^{1 *}$, Luis García-Vallejo², José Nuñez Gestal ${ }^{3}$, Pablo Concheiro Coello², \\ Francisco Blanco Antona1, Miguel Toledano Trincado', Javier Sánchez González ${ }^{1}$, \\ M. Luz Martín Esteban', L. Colao" ${ }^{4}$, Carlos Vaquero Puerta ${ }^{5}$, Miguel Carbajo ${ }^{6}$ \\ ${ }^{1}$ Surgery Department, Medina del Campo Hospital, Valladolid, Spain \\ ${ }^{2}$ Surgery Department, CHUS, Calle Rua de Ramón Baltar, Santiago de Compostela, Spain \\ ${ }^{3}$ Radiology Department, CHUS, Calle Rua de Ramón Baltar, Santiago de Compostela, Spain \\ ${ }^{4}$ Department of General and Digestive Surgery, Medina del Campo Hospital, Valladolid, Spain \\ ${ }^{5}$ Surgery Department, University of Valladolid, Valladolid, Spain \\ ${ }^{6}$ CEOAD, Valladolid, Spain \\ Email: *icmolmo@gmail.com
}

Received 10 July 2015; accepted 14 August 2015; published 17 August 2015

Copyright (C) 2015 by authors and Scientific Research Publishing Inc.

This work is licensed under the Creative Commons Attribution International License (CC BY).

http://creativecommons.org/licenses/by/4.0/

c) (i) Open Access

\begin{abstract}
Background and Objectives: The aim of this study is to evaluate the usefulness of Magnetic Resonance Imaging (MRI) for planning surgery and postoperative control of complex cases of abdominal wall hernia. Methods: Included in a prospective data base with more than 350 patients treated in two centers, a prospective study over ten patients with complex incisional hernias was designed. In all cases, preoperative diagnosis study and postoperative imaging control by MR Imaging were established. In the early and late follow-up controls, local and general complications were specifically noted and studied by MRI in each case. Results: The preoperative MRI was effective method for an adequate understanding of the defects and hernia sack content. In the postoperative control, the MRI was useful in the follow-up of the mesh integration, detection of complications and diagnosis of the relapsing. Conclusion: In our experience the MRI test is specially recommended in the preoperative workup of complex cases of incisional hernia.
\end{abstract}

\section{Keywords}

Incisional Hernia, MR Imaging, Laparoscopic Eventroplasty

\footnotetext{
"Corresponding author.
}

How to cite this paper: Martín del Olmo, J.C., et al. (2015) The Usefulness of Magnetic Resonance Imaging in the Preoperative Study and Postoperative Control in the Laparoscopic Treatment of the Incisional Hernia. Surgical Science, 6, $376-382$. http://dx.doi.org/10.4236/ss.2015.68055 


\section{Introduction}

The laparoscopic approach for the treatment of incisional hernias has been justified by several advantages compared to open surgery: it avoids aggression to the abdominal wall by laparotomy and unnecessary extensive dissections. It also eliminates the need to use tension sutures on the abdominal wall and the necessity of drains. The postoperative immobilization of the patient, hospitals stay and wound infections are fewer than in open surgery [1]-[3].

Moreover, the laparoscopic technique provides a complete exploration of the abdominal cavity, making the parietal and visceral adhesiolysis easier, which is a basic step to maintain the stability of the abdominal contents. This is paramount to avoid the chronic abdominal pain linked to the adhesions caused by the open procedures [4]. This minor incidence of local and general complications and shorter hospital stay has been reported in different studies [5]-[8].

Technological improvement, new laparoscopic tools and new biocompatible tissues for prosthesis, are playing an important role in the development of this type of surgery, which is receiving increasing support among surgeons and patients.

The CT (computed tomography) studies of the abdominal wall have been recommended for a better comprehension of the incisional hernia and the visceral contents of the sac, and the follow-up of technical results [5] [9]-[13].

However, in spite of that several studies have been focused in the use of postoperative MRI (Magnetic Resonance Imaging) [6] [14]-[16], preoperative MRI prior to laparoscopic treatment of incisional hernias is not routinely used, mainly in the management of complex cases, where the information about the abdominal wall anatomy and the abdominal content is very important before and after the surgery. The purpose of this study is to reach new research in this field.

\section{Materials and Methods}

For this ten patients with complex incisional hernias in different places of the abdominal wall were selected from our prospective database [1], which started in 1994 and currently includes more than 300 patients with laparoscopic abdominal hernia repair.

The patients were selected progressively between 2000 and 2005, in the two centers referred, under the following criteria: multiple previous abdominal surgery; relapsed incisional hernia; sub-occlusive problems and obesity.

This study was carried out under a human studies approved protocol and informed consent was obtained from each one.

The group was included in a prospective and protocolized study.

Before surgery all of the patients were studied by MRI and the images submitted to discussion between the surgical and radiological team.

All of the patients were operated by laparoscopic approach with the double crown technique (Protack, USS, Norwalk, CT, USA) and PTFE Dual Mesh Plus prosthesis WL Gore \& Assoc, Inc, Flagstaff, AZ, USA) as previously described [5].

After hospital discharge, the patients were sending to MRI control in the first, three, six and twelve months. No more controls were considered necessary and the study was finalized after 12 months.

The MRI studies were made with Siemens-Symphony Magnetom (1.5 T.) equipment. The sequences used in the evaluation of the abdominal wall were T1 IN-OPP FASE, T2 HASTE, and T2 HASTE FAT-SAT. All the sequences were made in axial, sagittal, and in some cases, in oblique view. Intravenous contrast was not employed in any case.

The average age of the patients was 62 (range 34 - 76), six women and four men. All the patients were ASA II/III. Obesity (BMI > 30) was present in eight patients. Six patients had one o more previous hernia repair. Five patients had abdominal wall defect between 5 and $10 \mathrm{~cm}$ in diameter and the other five larger than $10 \mathrm{~cm}$. No cases of meshes shrinkage were detected.

In the early and late follow-up, local and general complications were specifically noted and studied by MRI in each case with a follow-up of twelve months.

\section{Results}

Preoperative MRI was very effective in showing the abdominal wall defects, not only in the axial position but in 
the sagittal view, allowing an appropriate evaluation of the hernia sac content, especially when the small bowel or colon were included. The evaluation of the presence and the extent of abdominal adhesions are very useful in surgery planning.

However the most important usefulness of the preoperative study was the possibility to get an accurately outline of the abdominal wall in the defect area in order to make a more effective treatment (Figure 1).

Preoperative MRI suggested the presence of adhesions and major o minor adhesiolysis was necessary to perform in all cases, without associated morbidity.

In the postoperative period, the first month control was especially useful to manage the immediate postoperative seroma: position, amount and relation with the mesh. In the axial and sagittal views it is possible to see the seroma between the skin and the mesh, behind the mesh inside the abdominal cavity, or both (Figures 2-4). Also, this approach allows to determinate the correct position of the mesh, the absence of relapsing and the relation with the intestinal content.

The three and six months control showed the evolution of the seroma, the integration of the mesh and the possibility of occult relapsing (Figure 5 and Figure 6), giving a complete information of the abdominal wall.

Finally, the one-year postoperative control permits us to evaluate if the mesh integration through the abdominal wall is adequate and the presence or absence of mesh shrinkage.

On the other hand, we must summarize that the average surgical time, postoperative hospital stay, abdominal wall defect, hernia sack content and patch size were similar to our general data in a large series of patients [1] [5].

There were no complications related to the pneumoperitoneum, trocars placement, adhesions removing or mesh fixation. There were no infections or other parietal inflammatory data. Concomitant laparoscopic procedures were one cholecystectomy and one TAPP inguinal hernioplasty.

Three patients developed big-size seroma (30\%) (Figures 2-4), but evacuation was not necessary.

Regarding the late complications, a patient developed an occult relapse (Figure 5 and Figure 6), resolved by a new laparoscopic procedure.

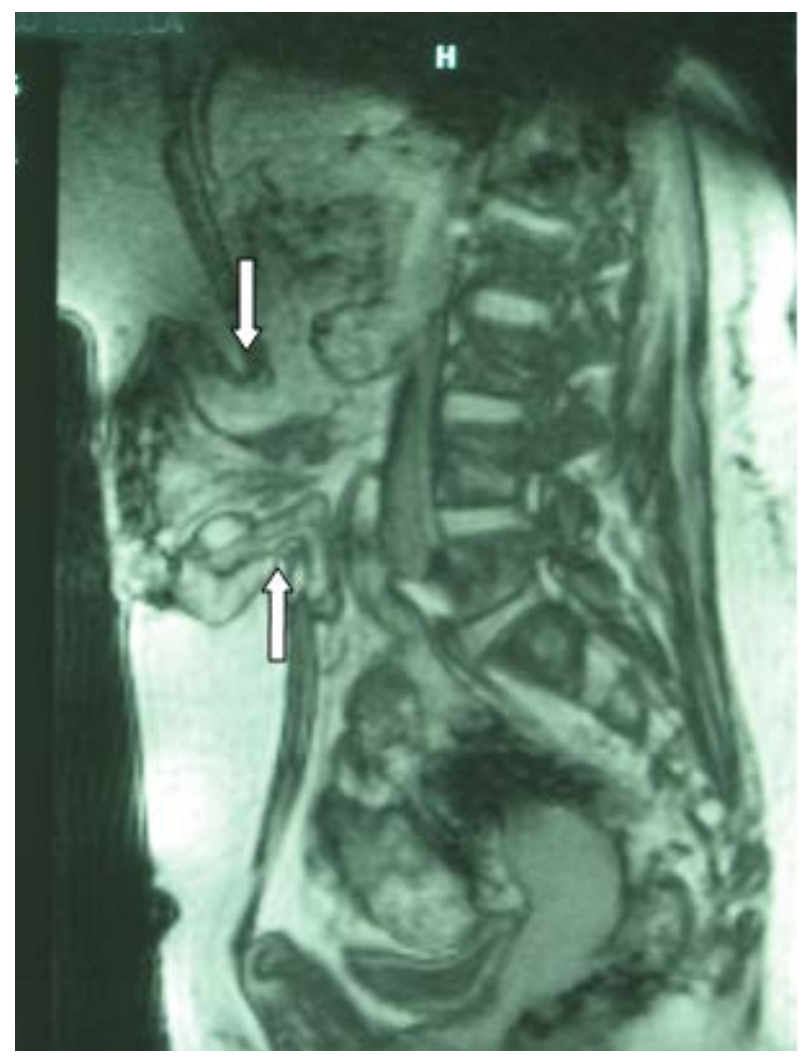

Figure 1. Preoperative MRI: sagittal view of a large incisional hernia. The arrows show the edges of the abdominal wall defect. 


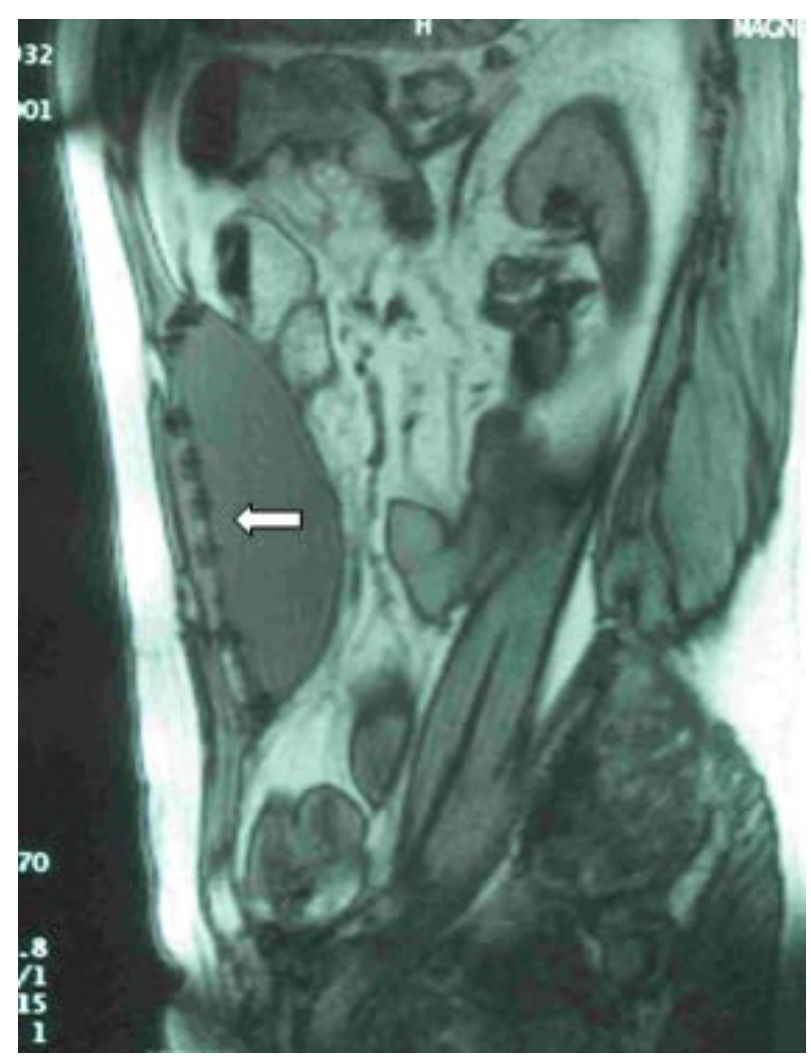

Figure 2. One month postoperative sagittal MRI: control after laparoscopic surgery on a central incisional hernia. The arrow shows the correct position of the mesh and tacks. A big seroma appears inside the abdominal cavity.

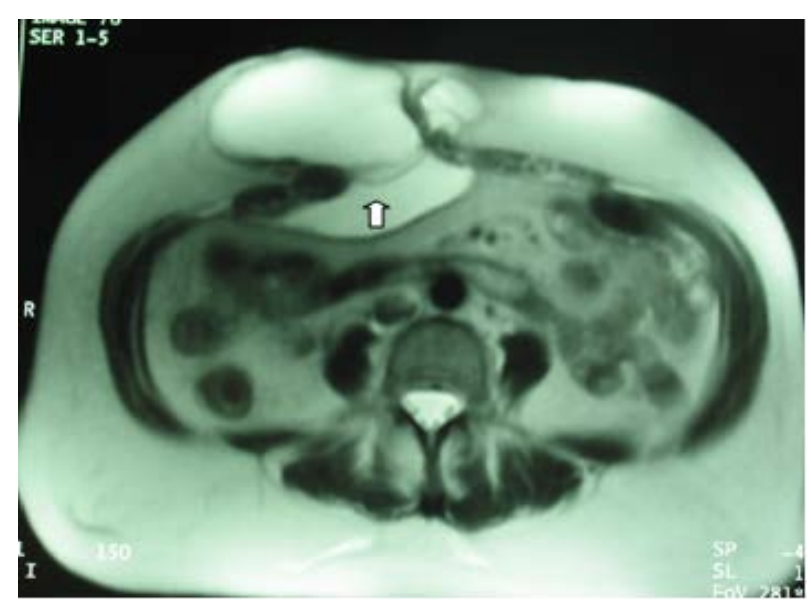

Figure 3. One month postoperative axial MRI control after laparoscopic surgery on a middle-right flank incisional hernia. The arrow shows the mesh. The seroma appears both under the skin and inside the abdominal cavity.

\section{Discussion}

Laparoscopic abdominal wall surgery is currently being developed due to its good results, which have widely surpassed the initial perspectives with this approach [17]. There are few comparative and randomized studies about this technique [1], so more clinical and comparative studies with evidence-based results are necessary [18]. 


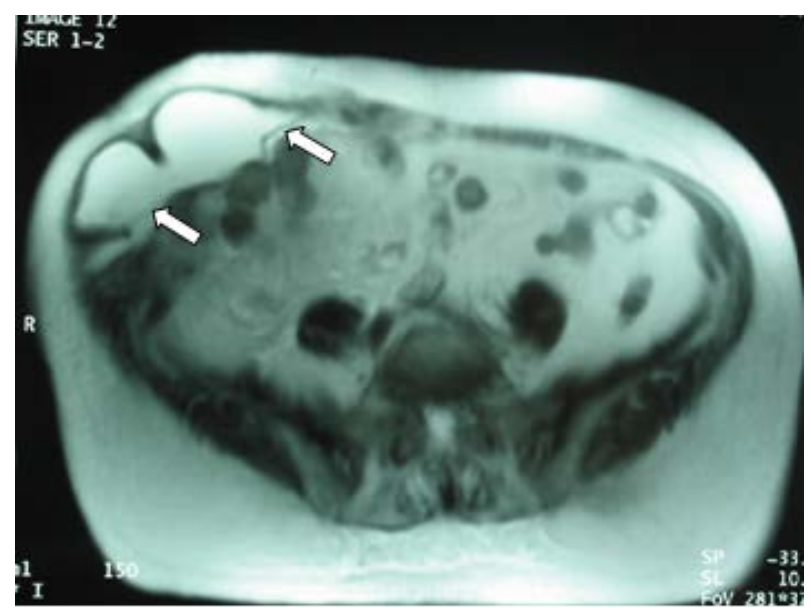

Figure 4. One month postoperative axial MRI control after laparoscopic surgery for a large right-subcostal incisional hernia. The arrows show the mesh and a big seroma under the skin.

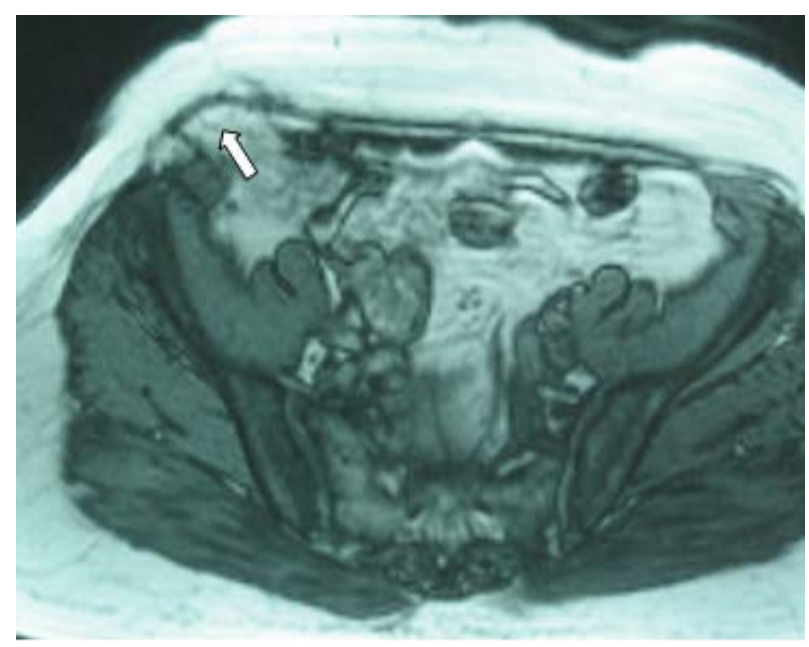

Figure 5. A six month postoperative axial MRI control after laparoscopic surgery for a large umbilical and right-side incisional hernia. In this view the arrow shows the mesh with evidence of a relapse.

In this sense our study seeks to provide a new concept in the imaging diagnosis and treatment of the laparoscopic abdominal wall hernias. It is true that the CT studies, both in the pre and postoperative course, have been useful [10] [11] and recommended [5], but only some MRI studies have been reported by the radiologists in the diagnosis of both inguinal and incisional hernias [4] [12] [19].

The MRI study is a quick procedure that avoids the necessity of a contrast dye or previous preparation for the patient. Besides, the MRI test does not emit any ionizing radiation like the CT studies.

The MRI images offer some advantages over the CT ones, allowing a wider possibility of radiological projections: axial, sagittal, coronal and oblique, giving important information for the surgeon.

In the preoperative study, the T1 sequences, offer better anatomical information of the abdominal wall and the sack content. However, the T2 sequences present better resolution in the postoperative period, especially in the valuation of the seroma.

The good quality of the images, the space resolution and the multi-view capacity are important features of this technique. The sagittal view is very useful for the surgeon, not only for the preoperative knowledge of the hernia anatomy, but for the diagnosed of complications and the final result of the procedure. 


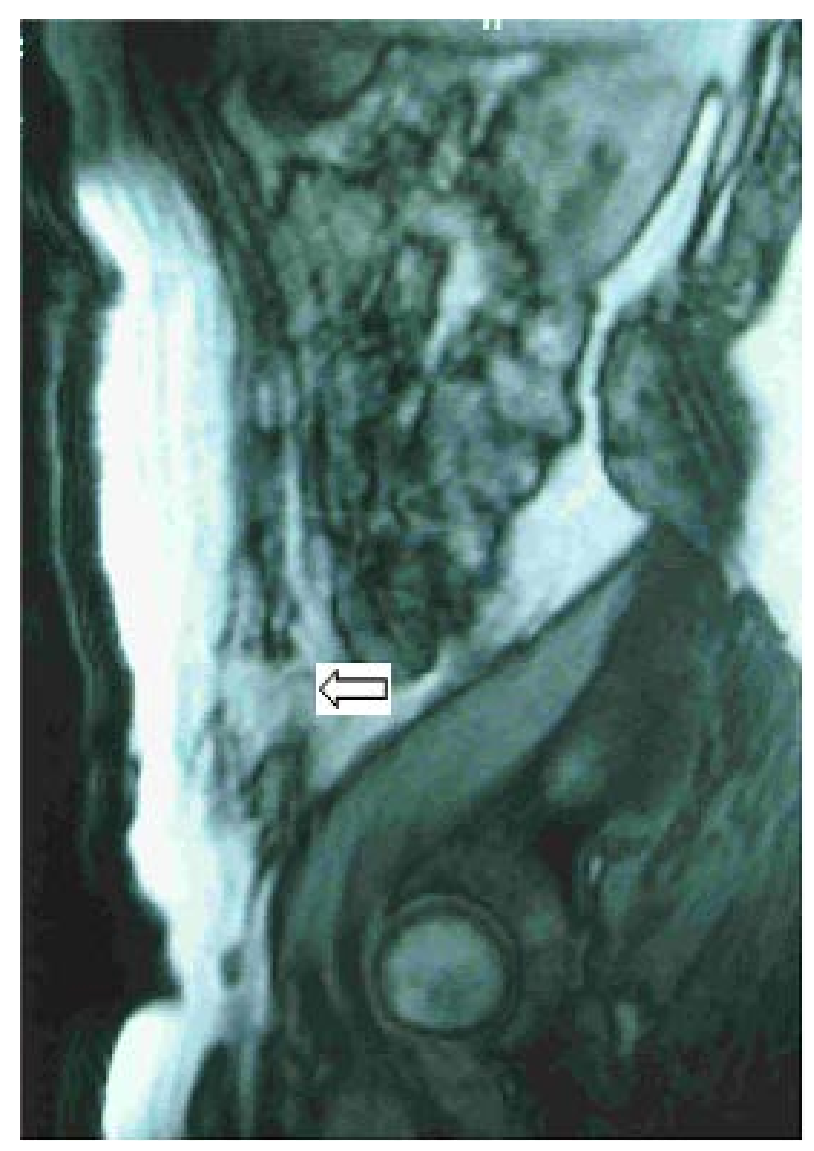

Figure 6. The same case of the Figure 5, in the sagittal view was possible to show perfectly an occult relapse in the right supra-iliac area.

We found preoperative MRI especially useful in planning the surgical approach. We usually use de contralateral approach for the lateral abdominal wall hernias but for midline hernias we work from the left side of the patient [5]. This approach would be changed to the right side depending of the presence and distribution of surgical adherences in the preoperative MRI, reducing the possibility of iatrogenic lesions of the bowel.

Obviously, this study is limited by the short number of cases and we don't use it routinely in all patients because of the costs. In our opinion this diagnostic tool should be reserved for complex cases, especially in the suspicion of severe abdominal adhesions.

\section{Conclusion}

In conclusion, the usefulness of the imaging MRI technique in the diagnosis and follow-up of the laparoscopic treatment of the incisional hernias resides in providing to the surgeon an appropriate tool for an adequate evaluation of anatomical abdominal wall defects before the surgical repair and an excellent evaluation of the complications related to it. These aspects are especially relevant in complex cases.

\section{References}

[1] Carbajo, M.A., Martín del Olmo, J.C., Blanco, J.I., et al. (1999) Laparoscopic Treatment vs. Open Surgery in the Solution of Major Incisional and Abdominal Hernias with Mesh. Surgical Endoscopy, 13, 250-252. http://dx.doi.org/10.1007/s004649900956

[2] Le Blanc, K.A. (2000) Current Considerations in Laparoscopic Incisional and Ventral Herniorraphy. JSLS, 4, 131-139.

[3] Park, A., Bird, D.W. and Lovrics, P. (1998) Laparoscopic and Open Incisional Hernia Repair: A Comparison Study. Surgery, 124, 816-822. http://dx.doi.org/10.1067/msy.1998.92102 
[4] Carbajo, M.A., Martín del Olmo, J.C., Blanco, J.I., et al. (2001) Therapeutic Value of Laparoscopic Adhesiolysis. Surgical Endoscopy, 15, 102. http://dx.doi.org/10.1007/s004640000275

[5] Carbajo, M.A., Martín del Olmo, J.C., Blanco, J.I., et al. (2003) Laparoscopic Approach to Incisional Hernia. Lessons Learned from 270 Patients over 8 Years. Surgical Endoscopy, 17, 118-122. http://dx.doi.org/10.1007/s00464-002-9079-0

[6] Kuehnert, N., Kraemer, N.A., Otto, J., et al. (2012) In Vivo MRI Visualization of Mesh Shrinkage Using Surgical Implants Loaded with Superparamagnetic Iron Oxides. Surgical Endoscopy, 26, 1468-1475. http://dx.doi.org/10.1007/s00464-011-2057-7

[7] Koehler, R.H. and Voeller, G. (1999) Recurrences in Laparoscopic Incisional Hernia Repairs: A Personal Series and Review of the Literature. JSLS, 3, 293-304.

[8] Toy, F.K., Bairley, R.W. and Carey, S. (1998) Prospective Multicenter Study of Laparoscopic Ventral Hernioplasty. Preliminary Results. Surgical Endoscopy, 12, 955-959. http://dx.doi.org/10.1007/s004649900755

[9] Donald, J.E. and Georges, A. (2003) CT Technique for Suspected Anterior Abdominal Wall Hernia. American Journal of Roentgenology, 181, 431-433. http://dx.doi.org/10.2214/ajr.181.2.1810431

[10] Gossios, K., Zikou, A., Vazakas, P., et al. (2003) Value of CT after Laparoscopic Repair of Postsurgical Ventral Hernia. Adom Imaging, 28, 99-102. http://dx.doi.org/10.1007/s00261-001-0156-y

[11] Lin, B.H., Vargish, T. and Dachman, A.H. (1999) CT Findings after Laparoscopic Repair of Ventral Hernia. American Journal of Roentgenology, 172, 389-392. http://dx.doi.org/10.2214/ajr.172.2.9930789

[12] Toms, A.P., Cash, C.C., Fernando, B., et al. (2002) Abdominal Wall Hernias: A Cross-Sectional Pictorial Review. Seminars in Ultrasound, CT and MRI, 23, 143-155. http://dx.doi.org/10.1016/S0887-2171(02)90001-6

[13] Schoenmaeckers, W., van der Valk, S.B., van den Hout, H.W., et al. (2009) Computed Tomographic Measurements of Mesh Shrinkage after Laparoscopic Ventral Incisional Hernia Repair with an Expanded Polytetrafluoroethylene Mesh. Surgical Endoscopy, 23, 1620-1623. http://dx.doi.org/10.1007/s00464-009-0500-9

[14] Fischer, T., Ladurner, R., Gangkofer, A., et al. (2007) Functional Cine MRI of the Abdomen for the Assessment of Implanted Synthetic Mesh in Patients after Incisional Hernia Repair: Initial Results. European Radiology, 17, 31233129. http://dx.doi.org/10.1007/s00330-007-0678-y

[15] Kirchhoff, S., Ladurner, R., Kirchhoff, C., et al. (2010) Detection of Recurrent Hernia and Intraabdominal Adhesions Following Incisional Hernia Repair: A Functional Cine MRI-Study. Abdom Imaging, 35, 224-231. http://dx.doi.org/10.1007/s00261-009-9505-z

[16] Kirchhoff, S., Lang, R., Kirchhoff, C., et al. (2008) Functional Cine MR Imaging for the Detection and Mapping of Intraabdominal Adhesions: Method and Surgical Correlation. European Radiology, 18, 1215-1223. http://dx.doi.org/10.1007/s00330-008-0881-5

[17] Forbes, S.S., Eskicioglu, C., McLeod, R.S., et al. (2009) Meta-Analysis of Randomized Controlled Trials Comparing Open and Laparoscopic Ventral and Incisional Hernia Repair with Mesh. British Journal of Surgery, 96, 851-858. http://dx.doi.org/10.1002/bjs.6668

[18] Sauerland, S., Walgenbach, M., Habermalz, B., et al. (2011) Laparoscopic versus Open Surgical Techniques for Ventral or Incisional Hernia Repair. Cochrane Database of Systematic Reviews, 2011, Article ID: CD007781. http://dx.doi.org/10.1002/14651858.cd007781.pub2

[19] Van den Berg, J.C., de Valois, J.C., Go, P.M., et al. (2000) Preoperative and Postoperative Assessment of Laparoscopic Inguinal Hernia Repair by Dynamic MRI. Investigative Radiology, 35, 695-698.

http://dx.doi.org/10.1097/00004424-200011000-00008 